\title{
TRANSVERSE PATTERNS IN TYPE-II OPTICAL PARAMETRIC OSCILLATORS
}

\author{
G. Izús ${ }^{[1, *]}$, M. Santagiustina ${ }^{[1,2]}$, M. San Miguel ${ }^{[1]}$, and P. Colet ${ }^{[1]}$ \\ [1] : Instituto Mediterráneo de Estudios Avanzados, IMEDEA (CSIC-UIB), \\ Universitat Illes Balears, E-07071 Palma de Mallorca, Spain. ${ }^{[+]}$ \\ [2] : INFM, Dipartimento di Elettronica ed Informatica, \\ Università di Padova, via Gradenigo 6/a, 35131 Padova, Italy.
}

\begin{abstract}
We study the effects produced by walk-off and cavity induced polarization coupling in the process of pattern formation in a type-II optical parametric oscillator (OPO). Walk-off generates a convectively unstable regime in which intensity patterns sustained by noise are produced. An absolute unstable regime is also present. In this regime a competition between two traveling waves of different wavelength takes place. The presence of a birefringent and/or dichroic mirror produces homogeneous phase locked solutions separated by Domain Walls similar to Bloch walls in magnetic systems. The spontaneous motion of these walls around point defects produces persistent spatio-temporal states.
\end{abstract}

\section{INTRODUCTION}

Pattern formation is an ubiquitous manifestation of nonlinearity which presents specially interesting features in nonlinear optical systems [1]. Among these features are phenomena linked to the vectorial degree of freedom associated with the polarization of light and to the macroscopic manifestations of quantum noise (disorder). A paradigm for these studies is the Optical Parametric Oscillator (OPO) [2] in which transverse patterns have been recently observed [3].

We consider transverse pattern formation in a type-II OPO: The nonlinear medium inside an optical cavity leads to frequency down conversion of the photons of a pump beam. The down-converted beams are polarized along the ordinary and extraordinary axes, i.e. are polarization non-degenerate. The anisotropy of the crystal can cause the extraordinary polarized component to walk-off the ordinary one in the plane transverse to the beam propagation direction. The resulting advectionlike phenomenon produces quantum noise amplification [4]. The bire- 
fringence/dichroism of the mirrors of the cavity leads to an additional interaction by way of a linear coupling between ordinary and extraordinary beams. Here, we discuss effects introduced in the process of pattern formation by: a) walk-off $[5,6]$ and b)linear polarization coupling [7].

\section{MEAN FIELD EQUATIONS FOR A TYPE-II OPO}

The equations that describe the time evolution in a phase-matched ring optical resonator filled with a birefringent, nonlinear quadratic medium, couple four complex slowly varying envelopes: the linearly polarized components $A_{0,0^{\prime}}(\vec{r}, t)$ of the intracavity Second Harmonic (SH) field pumped by $E_{0}$, and the signal $A_{1}(\vec{r}, t)$ (ordinary polarized) and idler $A_{2}(\vec{r}, t)$ (extraordinary polarized) components of the down converted Fundamental Harmonic (FH) field. We consider a mean-field approximation in which the longitudinal spatial dependence is averaged out. For the case of frequency degeneracy between signal and idler the equations obtained along the lines of the derivation in ref. [5] are

$$
\begin{aligned}
\partial_{t} A_{0^{\prime}}= & \gamma_{0^{\prime}}\left[-\left(1+i \Delta_{0^{\prime}}\right) A_{0^{\prime}}+i a_{0^{\prime}} \nabla^{2} A_{0^{\prime}}+c_{0^{\prime}} A_{0}\right]+\sqrt{\epsilon_{0^{\prime}}} \xi_{0^{\prime}}(\vec{r}, t) \\
\partial_{t} A_{0}= & \gamma_{0}\left[-\left(1+i \Delta_{0}\right) A_{0}+E_{0}+i a_{0} \nabla^{2} A_{0}+2 i K_{0} A_{1} A_{2}+c_{0} A_{0^{\prime}}\right]+ \\
& \sqrt{\epsilon_{0}} \xi_{0}(\vec{r}, t) \\
\partial_{t} A_{1}= & \gamma_{1}\left[-\left(1+i \Delta_{1}\right) A_{1}+i a_{1} \nabla^{2} A_{1}+i K_{0} A_{2}^{*} A_{0}+c_{1} A_{2}\right]+ \\
& \sqrt{\epsilon_{1}} \xi_{1}(\vec{r}, t) \\
\partial_{t} A_{2}= & \gamma_{2}\left[-\left(1+i \Delta_{2}\right) A_{2}+\rho \partial_{y} A_{2}+i a_{2} \nabla^{2} A_{2}+i K_{0} A_{1}^{*} A_{0}+c_{2} A_{1}\right]+ \\
& \sqrt{\epsilon_{2}} \xi_{2}(\vec{r}, t)
\end{aligned}
$$

Here $\vec{r}=(x, y)$ is the transverse spatial vector, $t$ is the time and $\gamma_{j}, \Delta_{j}$ and $a_{j}$ are respectively the effective cavity decay rate, effective mode detuning and diffraction for the field $A_{j}$. The nonlinear coefficient coupling is $K_{0}$ and $\rho$ is the walk-off coefficient. The linear coupling coefficients $c_{0,0^{\prime}}, c_{1,2}$ are related to the dichroism and to the birefringence of one of the mirrors of the ring cavity [7]. Such linear coupling of the linearly polarized components can also arise from an intracavity quarter wave plate in a standing wave cavity configuration [8]. The last term of each equation is an independent complex Gaussian white noise with zero mean value and delta-correlated in space and time. These noise terms describe quantum noise in the Wigner representation for the linearized version of eqs.(1) [9], but they can also account for thermal or input fluctuations. 


\section{WALK-OFF EFFECTS}

We first consider the simpler case in which there is no direct polarization coupling $\left(c_{0,0^{\prime}}, c_{1,2}=0, A_{0}^{\prime}=0\right)$ and study the effects of walk-off in the pattern forming OPO threshold. In this case eqs.(1) have the uniform steady state solution $A_{0}=E_{0} /\left(1+i \Delta_{0}\right), A_{1}=A_{2}=0$. A linear stability analysis determines that $A_{0}$ is stable while $A_{1}$ and $A_{2}^{*}$ become unstable if the normalized pump intensity $F=K_{0} E_{0} /\left(1+i \Delta_{0}\right)$ is such that:

$$
|F|^{2} \geq 1+\left[\frac{q^{2} \tilde{a}+\tilde{\Delta}+\gamma_{2} \rho q_{y}}{\gamma_{1}+\gamma_{2}}\right]^{2}
$$

At the critical threshold $|F|=\left|F_{c}\right|=1$ traveling waves $A_{1}, A_{2}^{*} \simeq$ $\exp [i \vec{q} \vec{r}+\lambda(\vec{q}) t]$ become unstable. The critical two-dimensional real wave-vectors $\vec{q}$ are on a circle centered at $\vec{q}_{0}=\left(0, q_{0, y}\right)$ with radius $R=\left|\vec{q}-\vec{q}_{0}\right|=\sqrt{q_{0, y}^{2}-\tilde{\Delta} / \tilde{a}}$, where $q_{0, y}=-\gamma_{2} \rho / 2 \tilde{a}, \tilde{a}=\gamma_{1} a_{1}+\gamma_{2} a_{2}$, and $\tilde{\Delta}=\gamma_{1} \Delta_{1}+\gamma_{2} \Delta_{2}$. When there is no walk-off the rings of the unstable modes for $A_{1}$ and $A_{2}$ overlap and, for $|F|>\left|F_{c}\right|$, any $\vec{q}$ mode on the ring, and the opposite for the orthogonal component, are selected by spontaneous symmetry breaking. Hence a phase pattern appears in $A_{1}$ and $A_{2}$, while the intensity in both polarizations remains homogeneous [10]. Pattern formation requires an instability at a finite wavenumber, which only occurs for $\tilde{\Delta}<0$ when $\rho=0$. Walk-off shifts the center of the rings along the direction of the advection term in opposite directions for $A_{1}$ and $A_{2}$, and it also increases their radius $R$. The shift appears on both rings because of conservation of photon momentum which has no transverse component in the SH pump. Walk-off also induces a drift of the pattern and breaks the rotational symmetry, so that the selected wave-vector orientation is parallel to the walk-off direction.

The transverse drift induced by the walk-off can overcome the spreading velocity of the unstable modes that form the pattern, so that a pattern forming perturbation grows but leaves the system: this is the convectively unstable regime, which is found for pump amplitudes larger than $\left|F_{c}\right|$ and up to an absolutely instability threshold $\left|F_{a}\right|$. This threshold can be determined by extending the linear analysis to complex wavevectors $\vec{k}[4,5,11]$.

At the threshold of absolute instability $|F|=\left|F_{a}\right|$ the same traveling wave for $A_{1}$ and $A_{2}^{*}$ is selected. It is oriented perpendicularly to the $y$-axis [6]. The wavelength is locally selected with equal probability between two possible ones. These two wavelengths correspond to the two wavevectors of the unstable ring along the two senses of the advection direction. These are the most unstable modes. For large enough 

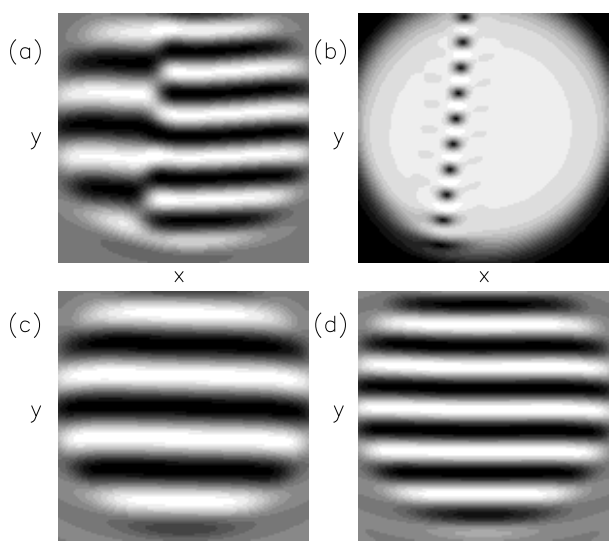

$x$

$\times$

Fig. 1. Transient competition between two wavelengths associated with the most unstable modes: a) $\Re\left(A_{1}\right)$, b) $\left|A_{1}\right|^{2}$; c) and d) show $\Re\left(A_{1}\right)$ for two possible final states where the largest or shortest wavelength structure has been selected. Parameters are $2 a_{0}=a_{1,2}=0.25, \gamma_{0,1,2}=1, \Delta_{0}=0, \Delta_{1,2}=$ $-0.15, K_{0}=1, \rho=0.15, \epsilon_{0,1,2}=0 ; E_{0}$ is a super-Gaussian beam of maximum amplitude $E_{\max }=1.05$.
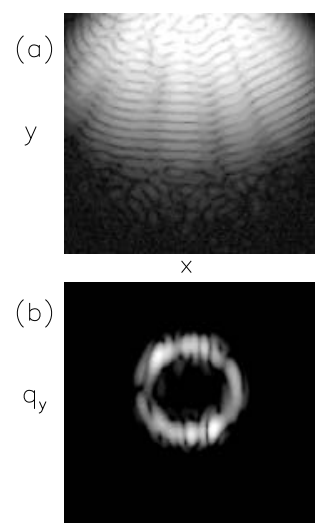

$q_{x}$

Fig. 2. Intensity of the signal field $A_{1}$ in the a) near field and b) far field, in the convectively unstable regime. $\mathrm{Pa}$ rameters as in fig. 1 except for $\epsilon_{0}=$ $\epsilon_{1}=\epsilon_{2}=2 \times 10^{-13}, \Delta_{1,2}=-0.2$ and $E_{\max }=1.009$.

systems a transient regime is observed in which a competition between these two wavelengths (phase stripe patterns) takes place as shown in Fig.1a. $\Re\left(A_{1}\right)$ shows stripes perpendicular to the drift direction while the intensity $\left|A_{1}\right|^{2}$ is homogeneous in each of the regions dominated by a given wavelength. The front between these regions of different wavelength displays a set of vertically ordered defects (Fig.1b). The field $A_{2}^{*}$ displays a pattern identical to that shown for $A_{1}$. For long times one of the two wavelengths fills the system, as shown in Fig.1c and Fig.1d.

In the convectively unstable regime, i.e., for $1<|F|<\left|F_{a}\right|$, noisesustained structures are expected. These structures, although advected away, are continuously regenerated by noise which at each space point excites all the unstable modes [5]. The modes of fastest growth, which are oriented along the advection direction, interfere generating intensity stripes as shown in the numerical solution of Fig.2a. The active modes in this regime can be seen in Fig.2.b (far field). The existence of intensity patterns is a new feature for a type-II OPO, which results from the interplay of the convective nature of the instability (i.e. the walk-off) and the presence of noise [6]. 


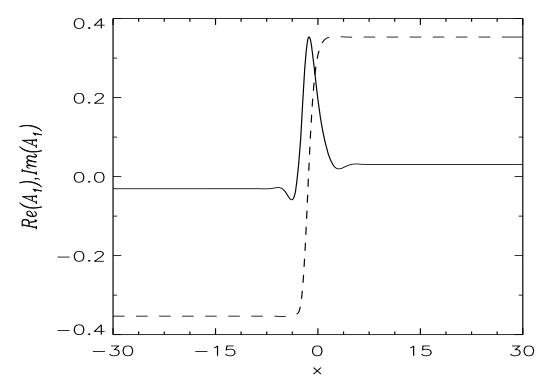

Fig.3 A Bloch wall obtained from a numerical solution of eqs.(1) in one spatial dimension. Solid line represents the real part of $A_{1}$ and the dotted line the imaginary one. Parameters are $\gamma_{0,1}=1, \gamma_{0^{\prime}, 2}=1.002, \Delta_{0,0^{\prime}}=0$, $\Delta_{1}=0.01, \Delta_{2}=0.03, a_{0,0^{\prime}}=0.125, a_{1,2}=$ $0.25, K_{0}=1, E_{0}=1.25, c_{0,0^{\prime}}=0.01(1+i)$ and $c_{1,2}=0.01$

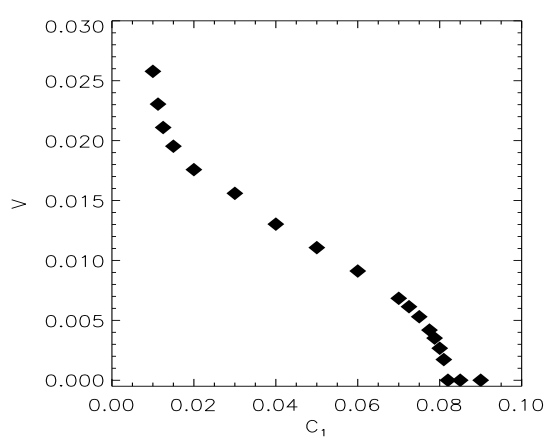

Fig. 4 Velocity of the Bloch walls as a function of $c_{1}$-real- in the regime $\gamma_{1} \Delta_{1} \neq \gamma_{2} \Delta_{2}$. Here $c_{1}=c_{2}, \gamma_{0,1}=1.001, \gamma_{0^{\prime}, 2}=1$, $\Delta_{0,0^{\prime}}=0, \Delta_{1}=0.01, \Delta_{2}=0.03, a_{0,0^{\prime}}=$ $0.125, a_{1,2}=0.25, K_{0}=1, E_{0}=1.25$ and $c_{0,0^{\prime}}=0.01(1+i)$.

\section{POLARIZATION COUPLING AND DOMAIN WALLS}

We now study the effects of birefringent-dichroic polarization coupling $\left(c_{0,0^{\prime}}, c_{1,2} \neq 0\right)$ neglecting for simplicity walk-off and noise $\left(\rho=\epsilon_{i}=0\right)$. A linear stability analysis shows that the trivial solution $A_{1,2}=0$, $A_{0}=\left(1+i \Delta_{0^{\prime}}\right) E_{0} /\left[1-\Delta_{0} \Delta_{0^{\prime}}-c_{0} c_{0^{\prime}}+i\left(\Delta_{0}+\Delta_{0^{\prime}}\right)\right], A_{0^{\prime}}=c_{0^{\prime}} E_{0} /[1-$ $\left.\Delta_{0} \Delta_{0^{\prime}}-c_{0} c_{0^{\prime}}+i\left(\Delta_{0}+\Delta_{0^{\prime}}\right)\right]$, is stable for $E_{0}<E_{c}$ where $E_{c}$ can be determined numerically. For $\widetilde{\Delta}>0$ homogeneous perturbations have the largest linear growth rate beyond threshold $E_{0}>E_{c}$. In this case two homogeneous solutions $A_{1,2}^{+}$and $A_{1,2}^{-}$of equal amplitude and phaseshifted by $\pi$ radiants $\left(A_{1,2}^{+}=-A_{1,2}^{-}\right)$bifurcate from $A_{1}=A_{2}=0$. They have the same probability to be selected at threshold. The existence of two phase-locked solutions is a consequence of the polarization coupling, since the relative phase of $A_{1}$ and $A_{2}$ is arbitrary when $c_{0,0^{\prime}}=c_{1,2}=0$. Numerical integrations of eqs.(1) confirm that stationary and uniform local domains, where $A_{1,2}$ are either $A_{1,2}^{+}$and $A_{1,2}^{-}$, form spontaneously. These domains of different but equivalent phase-locked solutions are separated by Domain Walls.

For small values of $c_{1,2}$ the Domain Walls are of the Bloch type, while for larger values they are of the Ising type [12]. In Fig.3 an example of a one-dimensional (1D) Bloch wall for $A_{1}$ is shown. The phase can rotate in two possible senses (chirality) across the interface, clockwise or 


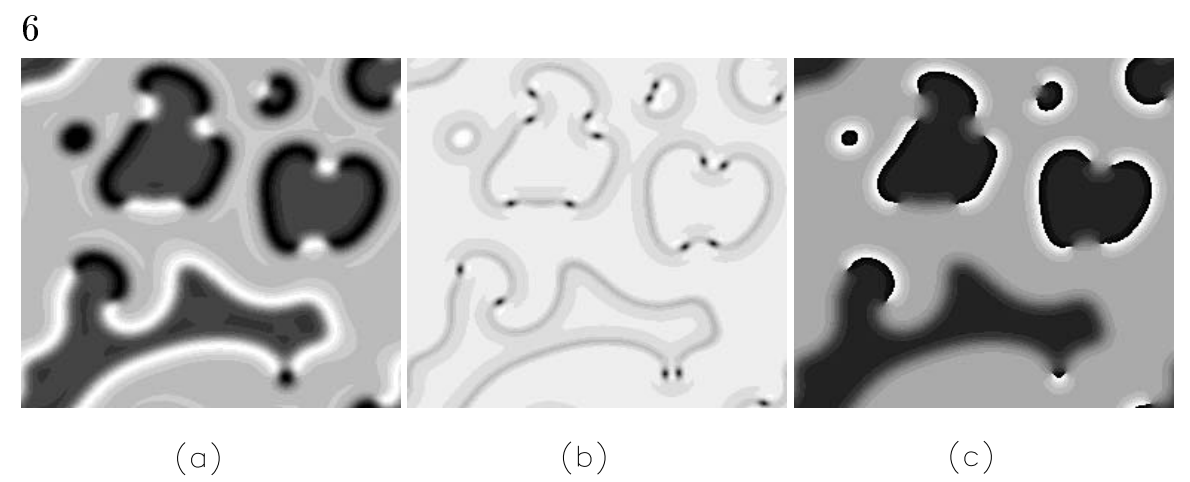

Fig.5. A snapshot at time $\mathrm{t}=1600$ of the field $A_{1}(x, y, t)$ obtained from a numerical integration of eqs.(1) with random initial conditions. a) real part; b) intensity and c) phase. Parameters are $\gamma_{0}=\gamma_{2}=1, \gamma_{0^{\prime}}=\gamma_{1}=1.002, \Delta_{0}=\Delta_{0^{\prime}}=0, \Delta_{1}=0.01$, $\Delta_{2}=0.03, E_{0}=1.25, a_{0}=a_{0^{\prime}}=0.125, a_{1}=a_{2}=0.25, K_{0}=1, c_{0}=c_{0^{\prime}}=0.025(1+i / 2)$ and $c_{1}=c_{2}=0.02(1+i)$.

counterclockwise in the complex plane. The wall for $A_{2}$ has the opposite chirality. The fields $A_{1,2}$ do not vanish at the core of the wall. On the contrary, on an Ising wall both the real and imaginary part of the field vanish at the same point in the core of the wall. The dynamics of $1 \mathrm{D}$ Bloch walls depends critically on the values of the cavity decay rate and the detuning. For $\gamma_{1} \Delta_{1}=\gamma_{2} \Delta_{2}$, 1D Bloch walls do not move. However, for $\gamma_{1} \Delta_{1} \neq \gamma_{2} \Delta_{2}$ walls of different chirality move in opposite directions. In Fig. 4 a plot of the velocity of a Bloch wall as a function of polarization coupling is shown. The point at which this velocity goes to zero identifies the transition point from Bloch to Ising walls.

In two dimensions (2D) domain walls are lines that locally can have opposite chirality. The change of chirality takes place in singular points, where the phase of the field is not defined and the amplitude goes to zero (defects), as we show in Fig.5. Observe, in Fig.5a, the domain walls that separate homogeneous regions of $A_{1,2}^{+}$from those of $A_{1,2}^{-}$; walls of different chirality are represented respectively by black or white thick curves. Defects associated with the changes of chirality appear as black dots in the intensity field (Fig.5b), while the phase field is shown in Fig.5c. The dynamics of Bloch walls in 2D depends also on the detuning and damping values, but it is also influenced by the curvature of the walls. For $\gamma_{1} \Delta_{1}=\gamma_{2} \Delta_{2}$ flat Bloch walls are stable. In this case the transient dynamics is mainly controlled by the curvature of the fronts. This leads to the growth of a phase at the expenses of the other, and to the annihilation of all the defects. For $\gamma_{1} \Delta_{1} \neq \gamma_{2} \Delta_{2}$ walls of different chirality move in opposite directions. Defects remain notably fixed and Bloch walls of different chirality tend to spiral around the defects. Fronts of equal chirality annihilate, when they collide, and new ones are 


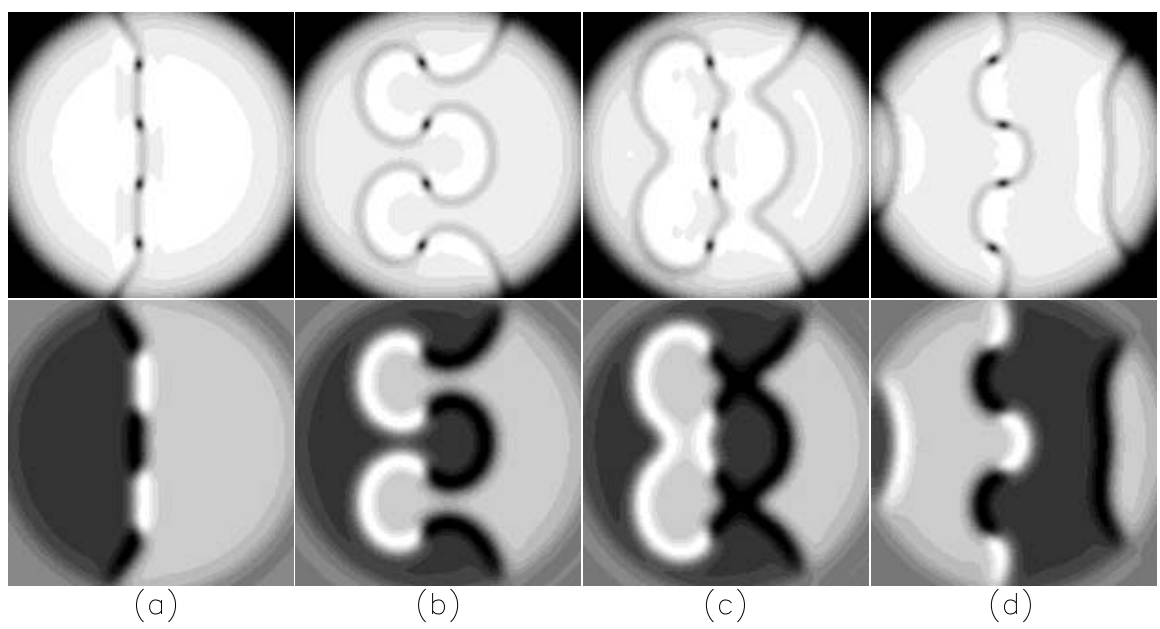

Fig.6. Time evolution generated from a Bloch wall with an array of defects. A succession of Bloch walls of opposite chirality are emitted: a) $t=0$; b) $t=1000$; $) t=1150$; $) t=1550$. Above: intensity patterns for $A_{1}$, below: the corresponding real part. Parameters are: $\gamma_{0}=\gamma_{2}=1, \gamma_{0^{\prime}}=\gamma_{1}=1.002, \Delta_{0}=\Delta_{0^{\prime}}=0, \Delta_{1}=0.01, \Delta_{2}=0.03, E_{0}=1.25$, $a_{0}=a_{0^{\prime}}=0.125, a_{1}=a_{2}=0.25, K_{0}=1, c_{0}=c_{0^{\prime}}=0.01$ and $c_{1}=c_{2}=0.025$.

generated by the defects (Fig.5). This leads to a persistent dynamical state and the system never reaches the state corresponding to one of the two homogeneous phase-locked solutions. An example of such persistent dynamical state, obtained from an initial condition consisting in a Bloch wall with segments of different chirality, is shown in Fig. 6.

\section{SUMMARY}

We have studied some polarization effects in the process of pattern formation in a type-II OPO. We have first considered the effect of walkoff, which induces the presence of absolutely and convectively unstable regimes. In the absolutely unstable regime phase stripes have been numerically observed, the wavelength being randomly selected out of two possible different values. The existence of two different values stems directly from the walk-off. An initial regime of wave-vector competition is also observed. We have also shown that in the convectively unstable regime intensity noise-sustained structures exist. These are a manifestation of quantum noise amplification.

Secondly, we have shown that signal/idler polarization coupling originated in a birefringent/dichroic cavity mirror leads to two equivalent phase locked states. Domain walls between these states can be Bloch 
walls with defects at points of change of chirality. The spontaneous motion of these walls sustains a persistent spatio-temporal pattern.

This work was supported by the European Commission through the Project QSTRUCT (ERB FMRX-CT96-0077). Financial support from Ministerio de Ciencia y Tecnología (Spain) Projects PB97-0141-02-C02 and BFM2000-1108 is also acknowledged.

\section{References}

[*] Permanent address: Departamento de Física, FCEyN, Universidad Nacional de Mar del Plata, Argentina. Member of CONICET (Argentine).

[+] http://www.imedea.uib.es/PhysDept

[1] Lugiato, L. A., Brambilla, M. and Gatti, A. (1999) Optical Pattern Formation, Advances in Atomic Molecular and Optical Physics, vol. 40, 229-306.

[2] Oppo, G-L., Brambilla, M., Lugiato, L. A. (1994) Formation and evolution of roll patterns in optical parametric oscillators, Phys. Rev. A 49, 2028-2032.

[3] Vaupel, M., Maitre, A., Fabre, C. (1999) Observation of Pattern Formation in Optical Parametric Oscillators Phys. Rev. Lett. 83, 5278-5281.

[4] Santagiustina, M., Colet, P., San Miguel, M. and Walgraef, D. (1997) Noisesustained convective structures in nonlinear optics, Phys. Rev. Lett. 79, 36333636.

[5] Santagiustina, M., Colet, P., San Miguel, M. and Walgraef, D. (1998) Twodimensional noise-sustained structures in optical parametric oscillators, Phys. Rev. E 58, 3843-3853.

[6] Izús, G., Santagiustina, M., San Miguel, M. and Colet, P. (1999) Pattern formation in the presence of walk-off for a type II optical parametric oscillator, J. Opt. Soc. Am. B 16, 1592-1596.

[7] Izús, G., Santagiustina, M. and San Miguel, M. (2000) Bloch Domain Walls in Type-II Optical Parametric Oscillators, Optics Letters 25, 1454-1456.

[8] Fabre, C., Mason, E. J. and Wong, N. C. (1999) Theoretical analysis of selfphase locking in a type-II phase matched optical parametric oscillator, Optics Communications 170, 299-307.

[9] Gatti, A., Wiedemann, H., Lugiato, L. A., Marzoli, I., Oppo, G. L. and Barnett, S. M. (1997) Langevin treatment of quantum fluctuations and optical patterns in optical parametric oscillator below threshold, Phys. Rev. A 56, 877-897.

[10] Longhi, S. (1996) Traveling-wave states and secondary instabilities in optical parametric oscillators, Phys. Rev. A 53, 4488-4499.

[11] Deissler, R. J. (1985) Noise-sustained structure, intermittency and the Guinzburg-Landau equation, J. Stat. Phys. 40, 371-395; (1989) External noise and the origin and dynamics of structure in convectively unstable systems, $J$. Stat. Phys. 54, 1459-1488.

[12] Coullet, P., Lega, J., Houchmanzadeh, B. and Lajzerowicz, J. (1990) Breaking Chirality in Nonequilibrium Systems, Phys. Rev. Lett. 65, 1352-1355. 